\title{
Litio y pueblos originarios: Desarrollo e identidad
}

\author{
Nicolás Handelsman ${ }^{1}$ \\ nicolas.handelsman@gmail.com \\ Rec. 14/05/2018 Apr. 22/08/2018
}

\begin{abstract}
Resumen
El presente analiza el conflicto que se está sucediendo en la actualidad, en torno a la explotación de yacimientos de litio en el noroeste argentino. El litio es un recurso estratégico para el desarrollo tecnológico, especialmente en lo que respecta a la llegada de los autos eléctricos. En este contexto, los pueblos originarios Kollas y Atacamas se ven amenazados ya que sus tierras ancestrales albergan el mayor yacimiento de litio del mundo ¿El Desarrollo justifica vulnerar las tradiciones de las minorías?
\end{abstract}

\begin{abstract}
This article analyzes the actual conflict related to the Lithium mines in the northwest area of Argentina. Lithium is a key resource for technologic development, especially regarding the implementation of the electric car. In this context, native population of the region is threatened because their lands are the world biggest lithium deposit. ¿Does development justifies harming traditions of minorities?
\end{abstract}

\section{Introducción}

¿Por qué la explotación de un recurso natural como el litio en la Argentina representa un dilema ético?

Para responder esta pregunta es importante dar un marco a la discusión, que permita visualizar los intereses en conflicto. Por un lado, el litio es uno de los recursos estratégicos centrales en el futuro cercano de la humanidad. Esto se debe a que es uno de los materiales más utilizados para la confección de baterías y por ende, se presenta como la llave para desarrollos tecnológicos como dispositivos móviles (celulares, tablets, etc.), autos eléctricos y también para el uso de fuentes de energía renovables sin intermitencias (se acumula energía solar en baterías para las noches, por ejemplo). 
Se calcula que el $68 \%$ de las reservas naturales de litio se encuentran en la frontera entre Chile, Bolivia y Argentina, con lo cual en dicha región se ha desarrollado un proceso geopolítico en el cual entran en conflicto diversos intereses. Este fenómeno alcanza dimensiones internacionales ya que las principales empresas interesadas en la explotación de dicho recurso son de origen extranjero.

El conflicto de esta situación se da porque en los salares donde se encuentran los yacimientos de litio, viven comunidades originarias, Kollas y Atacamas. La actividad minera afecta directamente al modo de vida de dichas comunidades, poniendo en conflicto los intereses del sector minero frente a las costumbres ancestrales de los pueblos originarios. Entre los principales efectos de la minería de litio se pueden nombrar el uso desmedido de agua potable, modificaciones en la fauna de la región, contaminación de napas, etc.

Por ende, el dilema ético que se plantea en el presente trabajo busca contrastar las ansias del desarrollo tecnológico enfrentado al respeto por las tradiciones y el territorio de los pobladores de las salinas grandes, quienes ven amenazada su subsistencia.

\section{El Litio en el NOA}

Argentina es el tercer país más rico en yacimientos de litio de todo el mundo, junto con China. Bolivia y Chile son los dos principales países. Además, por las características de los yacimientos, las tecnologías utilizables en los yacimientos de la puna (Chile, Bolivia y el NOA) resultan ser las de menor costo, lo cual potencia el interés por parte de los capitales extranjeros de conseguir las concesiones de explotación de dichos yacimientos.

\section{Gráfico 6: RESERVAS Y RECURSOS DE LITIO, PARTICIPACIÓN POR PAÍS}
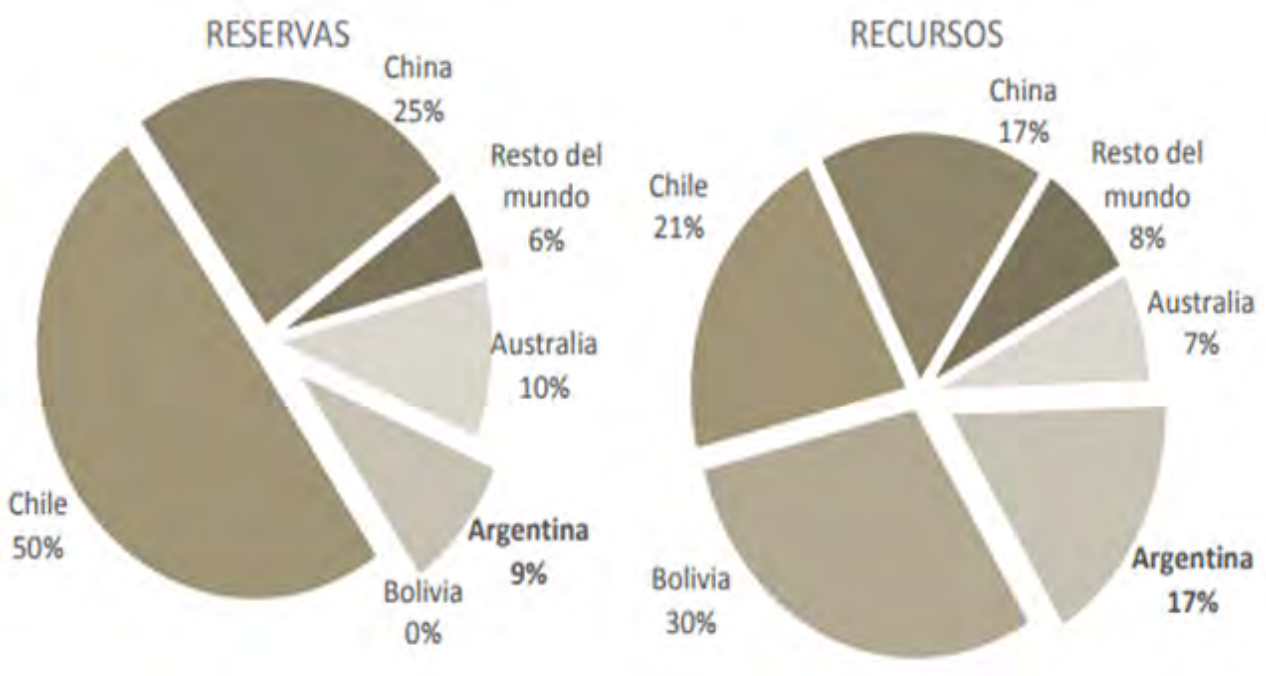
A su vez, otro factor que potencia el interés por la explotación del litio es la evolución del precio internacional del mineral, el cual presenta un crecimiento exponencial desde 2010 en adelante, y se pronostica que seguirá en ese sentido debido a la presión de la demanda internacional. Dicha demanda se proyecta que será cada vez mayor, en tanto se dé la inserción de los autos eléctricos y por la ampliación del uso de las baterías para dispositivos electrónicos y para acumulación de energía de fuentes renovables.

Gráfico 4: EVOLUCIÓN PRECIO ANUAL CARBONATO DE LITIO (USD POR TONELADA)

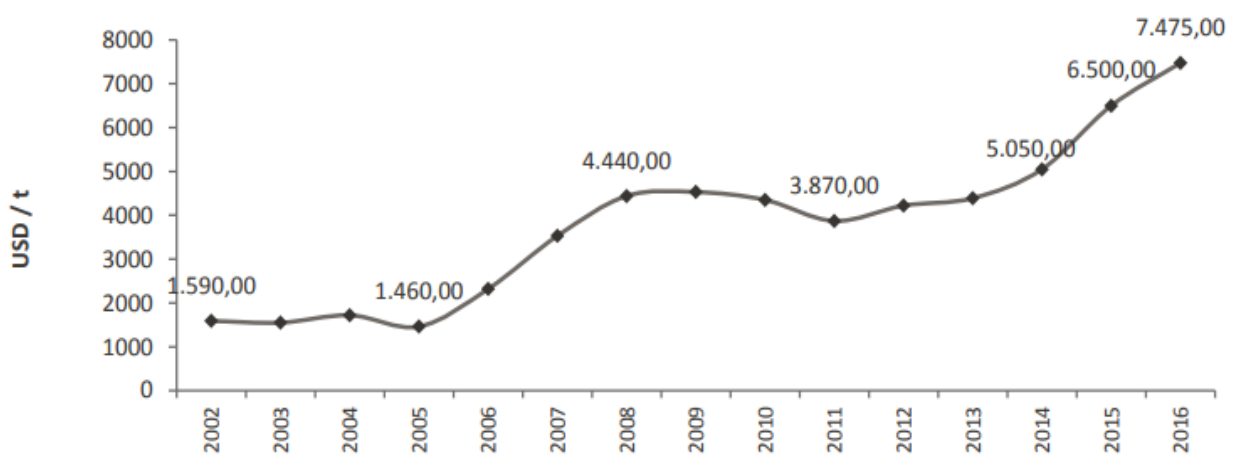

Fuente: Elaboración propia con base en USGS, AsianMetals y Metalary.com

Gráfico 11: DEMANDA PROYECTADA DE LITIO POR USO (2013-2025)

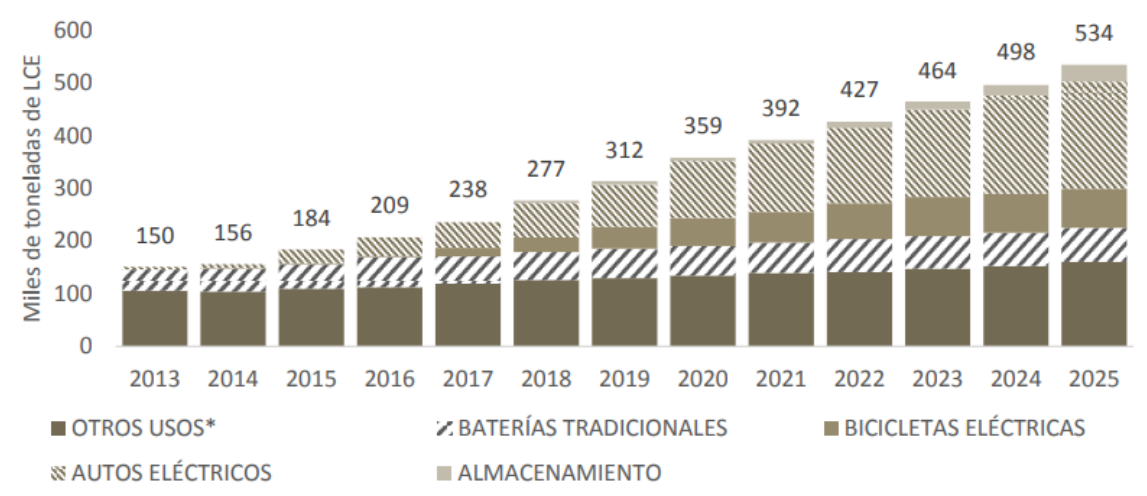

Fuente: Elaboración propia con base en Deutsche Bank | *Por otros usos no relacionados con baterías ver $\underline{\text { Gráfico } 3}$

Todos estos factores se configuran de manera tal que la región de la puna queda como foco de interés para los capitales extranjeros, los cuales entienden que el control económico del litio será estratégico para la configuración geopolítica de los próximos 20 años. Cabe destacar que en la actualidad, en Argentina hay solo 2 yacimientos de Litio en explotación (Salar de Olaroz y Salar del Hombre muerto), pero que ya están proyectados y en distintas etapa de avance más de 20 proyectos. Esto permite concluir que la intensidad de la actividad se incrementará a la brevedad, intensificando el conflicto con los pobladores originarios. 


\section{Los Pueblos Originarios}

Las Salinas Grandes se encuentran a 3.500 metros, y está conformado por los departamentos de Tumbaya y Cochinoca (Jujuy) y La Poma (Salta), a unos 120 kilómetros de Purmamarca (Jujuy). Un gran número de comunidades originarias habita la zona de la cuenca, tanto alrededor de la salina como hacia el norte, donde se ubica la laguna de Guayatayoc. Practican actividades agropecuarias de subsistencia, criando ganado caprino y cultivando maíz, arvejas, papas y otras hortalizas. Son poblaciones dispersas, donde las casas se ubican a gran distancia entre sí.

Las comunidades de Salinas Grandes mantienen relaciones con las diversas instancias del Estado y las empresas privadas, especialmente ofreciendo fuerza de trabajo con la producción de sal y el turismo. Los vínculos de estas comunidades con las salinas y el territorio son antiguos y vitales a la vez. Algunas de ellas, ubicadas a orillas mismas del salar, explotan la sal.

Los pobladores organizaron cooperativas de trabajo que explotan yacimientos de sal, la fraccionan y la ponen a la venta. Aquellos dedicados a la cría de ganado también utilizan sal para sus animales, que comercian con las comunidades cercanas a las salinas. Además, el pastoreo de llamas y demás animales, se realiza a través de un sistema de puestos rotativos, lo que hace que el territorio implicado en esta pequeña ganadería sea muy amplio.

Existen vínculos comerciales entre familias de estas comunidades. Generalmente se comercia con sal, o carne. También hay comercio con poblaciones de la quebrada o de zonas más selváticas, que producen algunas frutas y verduras imposibles de cosechar en la zona de salares. Se realizan ferias comunitarias y espacios para el trueque. Cada una de estas comunidades se organiza de manera particular. Tienen referentes locales, que muchas veces ocupan el cargo de presidente de la asociación civil a través de la que han obtenido una personería jurídica, y organizan asambleas de cada comunidad que funcionan como ámbitos de toma de decisiones.

Desde mediados de 2010 se creó la Mesa de Comunidades de la Cuenca de Guayatayoc y Salinas Grandes, donde treinta y tres comunidades se encuentran, debaten y organizan acciones a seguir, vigilando ante el interés por el litio de la región expresado por las empresas internacionales. Por lo tanto, estas poblaciones han desarrollado una instancia de organización "inter-comunitaria", que ha llevado a la Corte Suprema de Justicia la demanda a los Estados de Salta y Jujuy, por cuanto consideran las exploraciones mineras inconsultas y avasalladoras de sus derechos.

\section{Dilemas de la globalización y el litio: El rol de la ética}

La situación expuesta hasta aquí pone de manifiesto un conflicto de intereses entre diversos actores. Por un lado, el capital privado toma la bandera del progreso tecnológico, y del enriquecimiento privado, y para ello precisa disponer del territorio de los salares, y de su escasa agua potable. Por el otro lado, se observa a comunidades originarias organizadas, que reconocen el potencial negocio en su territorio ansestral y cómo este pone en riesgo la sustentabilidad de su modo de vida. 
Estas comunidades, a modo de resistencia solicitan ser consultadas previamente a la realización de cualquier proyecto de desarrollo, mediante un documento llamado "Huellas de Sal (Kachi Yupi)". Este pedido se apoya en un marco legal que tanto a nivel provincial, nacional e internacional, presenta normativas que promueven distintos derechos en favor de las comunidades originarias (Derecho a la libre determinación, derecho a la consulta previa, derecho a la tierra, derecho al agua, etc.). Por último, este documento propone una metodología detallada para realizar una consulta previa, y así validar los proyectos productivos a desarrollarse en el territorio de la Salinas Grandes.

Resulta interesante el hecho de que los pobladores originarios, lejos de aislarse en su cosmovisión, se han organizado desde 2010, se han informado y han conseguido representación legal ante el Estado y el capital privado. En adición a esto, los argumentos expuestos en los documentos oficiales emitidos por las comunidades no se centran en temáticas religiosas o sobre deidades (La Pachamama, por ejemplo), sino que sin abandonar esas creencias manifiestan que el conflicto es de índole ético. Lo que se pone en juego con los proyectos mineros es la supervivencia de la cultura de la región, la vida de sus pobladores que son tan humanos como los accionistas de las empresas mineras.

[...] durante el año pasado, con preocupación, comenzamos a ser testigos mudos de una nueva actividad en la región, nadie nos informó nada a pesar de la vigencia de derechos que exigen que nos consulten (Constitución Nacional, Artículo 6 del Convenio 169 de la OIT). Nos enteramos por rumores y por los medios de prensa, que habían descubierto litio en el subsuelo de los salares de la Puna de Salta y Jujuy. Todos los anuncios del Gobierno, daban cuenta de la importancia de este nuevo emprendimiento para la economía nacional e internacional. Sin embargo, nadie dijo nada de cómo esta nueva explotación nos puede afectar a las comunidades y a nuestro territorio: a las salinas, a las vertientes de agua, a los pastos, a nuestros ganados, a la Pacha, a nuestras costumbres y creencias, etc. En síntesis: a toda nuestra vida [...] (Kachi Yupi, Huellas de Sal, 2015, p.13)

El rol que cumple la ética en este conflicto responde, en términos de Ricardo Maliandi, al de "prótesis". Esta "prótesis" opera como un mecanismo de compensación ante la falta de conciencia de los responsables de las empresas mineras, y del poder ejecutivo respecto a la existencia de un grupo de congéneres, los pueblos originarios, que gozan de los mismos derechos que empresarios y funcionarios públicos.

La ética debe ser el motor que movilice mecanismos de protección para los hombres y mujeres que se ven amenazados por sus pares, los responsables del negocio del litio. Estas personas no parecieran tener entre sus escalas de valores el respeto por la vida de los pobladores de las salinas.

La ética, como prótesis que inhibe la capacidad ofensiva de las personas, ha desarrollado a lo largo de la historia una batería de normativa legal que respalda y ampara los derechos de las comunidades que son invizibilizados y amenazados por fenómenos de la globalización que no reparan en las consecuencias ecológicas de sus actos. Organismos internacionales como la ONU o la OIT han redactado acuerdos, que tienen jerarquía 
constitucional, en los cuales se ponen de manifiesto en forma clara la necesidad de poner a reguardo los derechos que gozan las poblaciones originarias.

Estas iniciativas internacionales están fundamentadas en aspectos de ética cordial, basada en la empatía entendida como la capacidad de ponerse en el lugar del otro, de tomar como propias las injusticias ajenas. Sin embargo, la conducta de los empresarios y funcionarios públicos no es carente de ética. Existen argumentos racionales en favor de la explotación del litio.

Además de ser un negocio importante, el litio permite entre otras cosas reducir la dependencia de los combustibles fósiles, y potenciar el uso de las fuentes renovables de energía. Este tipo de argumentos permiten construir una ética racional en la cual se ponen en igual nivel la supervivencia de una comunidad con el avance tecnológico del uso de baterías sin así perder su lógica interna.

Un posible argumento en favor de la explotación del litio, basado en una ética racional, podría ser que mediante las baterías fabricadas con litio, se podría reducir la emisión de gases de efecto invernadero, que atentan contra la especie humana. Esto se debe a que se reemplaza la generación eléctrica con combustibles, por el uso de energía solar o eólica, que se acumule para cuando haya falta de viento o radiación solar. Así, la reducción de emisiones se presenta como un beneficio para la humanidad en su conjunto y las comunidades originarias afectadas resultan el mal menor dentro de esta situación.

De esta manera se plantea una solución unilateral, en la cual se asume el costo social de avanzar sobre los derechos de las comunidades originarias. Se acepta como tolerable ese mal, ya reconocido como un efecto negativo, del avance tecnológico que promete un beneficio global al bajar las emisiones, pero también representa un negocio para un sector de elites. Esta tolerancia se expresa como algo pasivo, que simplemente ve de lejos cómo suceden los fenómenos sin intervenir activamente.

Una visión alternativa a este problema podría ser tomar una actitud de tolerancia activa en donde se valorice la diversidad cultural y la protección de costumbres ancestrales de las comunidades originarias, y que se exprese la necesidad de llegar a formas de explotación del litio, de avance tecnológico, que no descuiden o ignoren la voz de los pueblos afectados. Buscar formas de compensación para apalear las externalidades negativas de la explotación minera.

Un ejemplo concreto de esto se da en Bolivia, otro país central en lo que respecta a las reservas de litio. Allí, la empresa estatal COMIBOL desarrollo una planta piloto de producción de litio en el Salar de Rio Grande de Lipez, en la cual se tomaron diversas medidas para minimizar el impacto ambiental de la actividad económica. Por ejemplo, se minimizó el uso de agua potable, reemplazada por agua salada del río, también se realizaron obras de protección adicional para evitar la contaminación de napas y se utilizaron todas piezas móviles para infraestructura, garantizando así que en el largo plazo el paisaje no se verá afectado.

Todas estas medidas implican un costo adicional para la actividad minera, y por ende atentan contra uno de los intereses principales de las empresas privadas, y por desgracia 
de muchos funcionaros públicos también: La ganancia. Lejos de defender la bandera del avance tecnológico y la reducción de emisiones de gases de efecto invernadero, la forma en que está avanzando la minería de litio en el NOA antepone la maximización de la ganancia empresaria antes que la preservación de la diversidad cultural y el respeto por la historia de los pueblos originarios.

Sin embargo, conseguir que los interlocutores reconozcan como mejor el argumento que satisface intereses universalizarles no depende sólo de la lógica interna del argumento, sino también - y en muy buena medida - de que los interlocutores estén predispuestos a interpretar correctamente cuáles son esos intereses, a buscar "desprevenidamente" las opciones más justas. A. Cortina; "Ethica Cordis; Isegoria No37, Julio-Diciembre 2007

\section{La sociedad del riesgo y la cosmovisión andina}

En el manifiesto "Huellas de Sal" publicado por las comunidades de Salinas Grandes, se expresan algunas de las premisas que guían el modo de vida de estas personas. Entre las que se destacan, se pueden nombrar el respeto por la naturaleza, el cuidado de explotar de manera sostenible los recursos naturales del salar y, por sobre todo, la idea de pensar de manera atemporal o sostenible en el tiempo la existencia de su cultura y su comunidad.

El presente documento contiene el trabajo en los territorios de las comunidades de la Cuenca de Salinas Grandes y Laguna de Guayatayoc, que organizadas construimos así, un sendero de lucha y de acción política con nuestra vida, en un escenario de disputa con los modelos y las políticas funcionales al despojo, en un horizonte por el Buen Vivir "Sumak Kawsay", en el que nuestras acciones son propuestas como herramientas, estrategias, producción y revalorización de saberes y conocimientos ancestrales. Son alternativas político-organizativas que tienen su grandeza en la realización comunitaria y su potencia en la perseverancia. Distinto a la realidad que se nos presenta en modelos y planes de desarrollo que no dialogan con lo que somos, que no dan cuenta de nuestra historia comunitaria, nuestras necesidades y potencialidades, y en los que nuestra voz no ha sido escuchada. (Kachi Yupi, Huellas de Sal, 2015, p. 6)

Ante esto, se opone otra visión opuesta, el de la sociedad industrial, que lejos de perseguir objetivos de perdurar y permanecer de manera sostenible, se enfoca en la obtención de ganancias de corto plazo mediante actividades extractivistas que ponen en jaque la resiliencia de los ecosistemas y las sostenibilidad de dicha actividad en el tiempo. Los responsables de las empresas encargadas de la actividad minera, son gerentes que viven a miles de kilómetros de la zona e influencia de la extracción del litio. Para ellos, estos proyectos son solo parte de un portfolio de inversiones que prometen ser muy redituables en el corto plazo, y posicionar de manera estratégica al sector dentro de la matriz energética mundial.

Es así como la globalización permite que los decisores puedan inhibir todo intento de solidaridad con los afectados de sus proyectos de inversión. Es tanta la distancia geográ- 
fica, cultural y de intereses que se vuelve muy dificultoso que se despierte una conciencia solidaria que atenué los efectos de la actividad desarrollada por el capital privado.

Distinto es el caso de los funcionarios públicos que actúan como cómplices el desarrollo de estas actividades extractivistas que atentan contra la ecología y la diversidad cultural del territorio sobre el cual gobiernan. En estos casos el motivo principal suele ser el enriquecimiento personal, dado que el capital privado se abre camino entre las instituciones públicas mediante sobornos o cabildeo (lobby). Así, los funcionarios que sí son conscientes de la existencia de comunidades, a las cuales ellos también representan, ignoran esta situación y permiten el desarrollo de actividades que van en contra de los derechos de esas personas, sin siquiera informarlo o bien consultar previamente para poder planificar acciones que remedien los perjuicios.

\section{Conclusiones}

El conflicto por la explotación del litio tiene diversos enfoques, que fueron expuestos en el presente trabajo. Por un lado, desde el ámbito legal, existe legislación a toda escala (desde internacional hasta provincial) que pone de manifiesto los derechos que gozan los pueblos originarios. Sin embargo, en la práctica, mediante artimañas legales, o simplemente ignorando las normas, la explotación del litio sigue avanzando al ritmo deseado por los inversores privados involucrados.

Esta presión desde el sector capitalista se da en un contexto de globalización, que tińe todo de un manto de insensibilidad ante las consecuencias de los actos. Lo único que no queda librado al azar, y que es motivo de profundos estudios y análisis es la rentabilidad de las actividades. El capital privado globalizado opera mediante ese parámetro, la búsqueda de la ganancia. Para eso los líderes de las empresas mineras si son activos y consientes. Por el contrario, la globalización logra cubrir o camuflar todos los efectos directos del desarrollo de actividades económicas, con la complicidad de las elites gobernantes, quienes recién un beneficio residual de la actividad y así pueden sostener estructuras políticas de dominación en el territorio, que requieren la complicidad y financiamiento del capital privado.

En el marco de la globalización, será necesario pues desarrollar una ética solidaria que rompa con los mecanismos de camullaje que el mismo sistema económico pone en funcionamiento para poder avanzar sobre los derechos de las minorías y sobre el medio ambiente. Esto requerirá desarrollos teóricos, instituciones activas y el compromiso de la sociedad civil, dado que el alcance del poder económico es tan vasto que no podrá ser fácilmente contrarrestado.

\section{Bibliografía}

- Ambrosini, C. "Responsabilidad en la Sociedad del Riesgo. Cenando sobre el Costa Concordia”. Material de Cátedra en la materia Ética de la gestión energética, Maestría en Gestión de la energía, UNLa, (inédito) 
— Ambrosini, C. (2011).“Ética profesional. La ética como mecanismo compensatorio en el mundo globalizado". Perspectivas metodológicas, (11), pp. 23-38.

- Delbuono, V. Tay Such, Toledo, E., Jerez,D., Mercado del Litio (2017). Situación actual y perspectivas, Informe Especial, Ministerio de Energía de la Nación. Disponible en http://cima.minem.gob.ar/assets/datasets/marzo_2017_-_informe_especial_litio_.pdf

- Fornillo, B. (2015). Geopolitica del Litio, Industria, Ciencia y Energía en Argentina. Buenos Aires: Editorial El Colectivo, CLACSO.

- Kachi Yupi - Huellas de la sal (2015). Procedimiento de consulta y consentimiento previo, libre e informado para las comunidades indígenas de las Salinas Grandes y Laguna de Guayatayoc. Disponible en http://naturaljustice.org/ wp-content/uploads/2015/12/Kachi-Yupi-Huellas.pdf

— Ströbele-Gregor J. (2009). “El Litio en Bolivia”. Petropress (13), Centro de Documentación e Información Bolivia (CEDIB). Disponible en http://www.desigualdades.net/Resources/Working_Paper/14_WP_Str_bele_Gregor_online_sp.pdf 